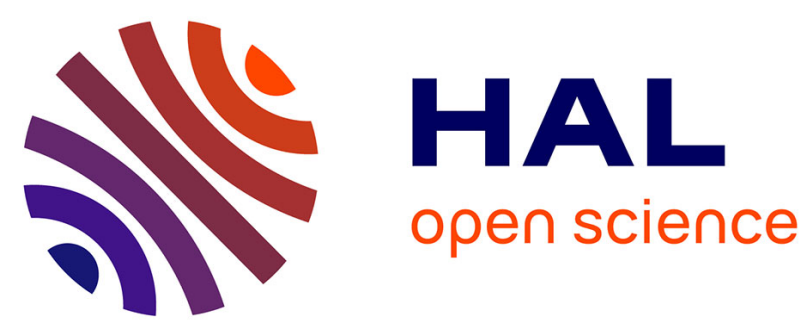

\title{
Development, presentation and tests of a hybrid thermal vibrational energy harvester based on lead free piezoelectric material \\ Neetu Kumari, Micky Rakotondrabe
}

\section{- To cite this version:}

Neetu Kumari, Micky Rakotondrabe. Development, presentation and tests of a hybrid thermal vibrational energy harvester based on lead free piezoelectric material. 2nd International conference on Electronics, Control, Optimization and Computer Science (ICECOCS 2020), Dec 2020, Kenitra, Morocco. 10.1109/ICECOCS50124.2020.9314542 . hal-03186544

\author{
HAL Id: hal-03186544 \\ https://hal.science/hal-03186544
}

Submitted on 31 Mar 2021

HAL is a multi-disciplinary open access archive for the deposit and dissemination of scientific research documents, whether they are published or not. The documents may come from teaching and research institutions in France or abroad, or from public or private research centers.
L'archive ouverte pluridisciplinaire $\mathbf{H A L}$, est destinée au dépôt et à la diffusion de documents scientifiques de niveau recherche, publiés ou non, émanant des établissements d'enseignement et de recherche français ou étrangers, des laboratoires publics ou privés. 


\title{
Development, presentation and tests of a hybrid thermal vibrational energy harvester based on lead free piezoelectric material
}

\author{
Neetu KUMARI ${ }^{1}$, Micky RAKOTONDRABE ${ }^{2, *}$ \\ 1: Automatic Control and Micromechatronic Systems department \\ FEMTO-ST Institute, CNRS - University Bourgogne Franche-Comté \\ Besançon, FRANCE \\ ${ }^{2}$ : Laboratoire Génie de Production \\ National School of Engineering in Tarbes (ENIT - INPT), University of Toulouse \\ Tarbes, FRANCE \\ ": mrakoton@enit.fr (corresponding author)
}

\begin{abstract}
Piezoelectric and pyroelectric effects are well known and have been widely used in the field of energy harvesting, actuators or sensors. This paper presents a cantilever structure based on a lead free piezoelectric-pyroelectric hybrid transducer based on PVDF (Polyvinylidene fluoride) piezoelectric, a Lithium Niobate piezoelectric, and a SMA (Shape Memory Alloy) materials which is capable of simultaneously harvesting mechanical and thermal energies. The piezoelectric cantilever has been studied using finite element modeling for application in self-powered sensors in automobiles. The cantilever are able to resonate under $300 \mathrm{~Hz}$ of frequency range. We calculated the output voltage from both the thermal and vibrational excitations with the help of Comsol Multiphysics finite-element software. We shows that the configuration in which the PVDF is the top layer, then SMA as the middle layer and Lithium niobate as the bottom layer has the highest value of harvested energy with an output voltage up to $12 \mathrm{Vpp}$.
\end{abstract}

Keywords-energy harvesting, hybrid, piezoelectric, and Shape Memory Effects, thermal.

\section{INTRODUCTION}

Energy harvesting is the process by which energy is derived from external sources (also known as ambient energy, e.g.: solar power, thermal energy, wind energy, salinity gradients, and kinetic energy), captured, and stored. Micro and nano-energy harvesting systems provide small amount of power that can be used in low-energy electronics and for small, wireless autonomous devices, like those used in wearable electronics and wireless sensor networks [1]. Despite the fact that the amount of power is small in these, they are becoming very popular because they are based on the green energy which in any way will not affect the environment. There are abundance of ambient micro and nano-energy which can be harvested: temperature gradients, temperature variation, electromagnetic energy due to radio and television broadcasting, mechanical vibrations, etc. A lot of research works have been done in the fields of micro or nano-energy harvesting systems (harvesters) to power devices with small consumptions. As a result, the devices are being optimized to require less and less energy, down to Pico joules for some applications [2], while the harvesting systems are now able to generate energies up to the milli joules range [3]. Nowadays, micro and nano-energy harvesting systems are starting to offer a real alternative over battery-powered solutions, with no maintenance needed and having very little adverse environmental effects.

Regarding mechanical micro energy, one of the most used principle to convert it intro electrical energy is exploiting the piezoelectric effect. Whilst this effect is already well known for sensing devices development [4] and self-sensing technique in piezoelectric actuators [5], a paramount of works exist in piezoelectric approach based mechanical energy harvesting. On the other hand, thermal sources which are widely available in the environment, energy can be directly converted into electricity by means of thermoelectricity (Seebeck effect) or pyroelectricity. To set up thermoelectric and pyroelectric power generators, significant spatial- and time-dependent temperature variations respectively are required. In turn, they also require optimized cold source management systems. The cold source usually consists in a compact sized radiator of which size and shape can become problematic at small scales. On the other hand there is another strong limitation from the low voltage or current delivered by these generators, which require complex and power-consuming buck-boost converters. This can be overcome by indirectly convert heat into electricity through mechanical transformation such as using smart composite structures. Such structures require two materials of different thermal properties and being mechanically coupled. The basic example of such composite is the bimetal structure. In this paper, we will present a composite structure for thermal energy harvesting. Not only the suggested harvester is capable of converting thermal energy through pyroelectricity but it can also convert mechanical vibrational energy by using piezoelectric effect. It will be called hybrid energy harvesting system in the sequel.

As the name suggests, hybrid energy harvesting systems can be defined as those which simultaneously harvest numerous energies of various natures at the same time by utilizing an integrated system [6]. By harvesting different types of energy through a single harvesting system will compensate for the performance of each individual system and will sum the various energies harvested from one single element $[7,8,9,10]$. The advantages of hybrid harvesting using one single system is probably the low production cost and the small sizes features contrary to the harvesting of several energies with several systems [11]. In counterpart, designing a hybrid harvester requires specific materials and 
appropriate design. In [11], a flexible hybrid energy harvester for simultaneously/individually harvesting heat, vibration, and solar energies was described. On the other hand, Dukhyun Choi [12] proposed flexible hybrid Nano architecture that can be used as both an energy scavenger and a touch sensor on a single system. The hybrid harvester had a total thickness of less than $500 \mathrm{~nm}$ on a plastic substrate and can provide electrical energy from both solar ray and vibrational touching energies. Yonas Tadesse et al. [13] presented a hybrid energy scavenging system that works with the uses electromagnetic and piezoelectric techniques. The system consists of piezoelectric crystals attached to a cantilever beam. The tip of the cantilever has an attached permanent magnet which vibrates within a coil fixed to the top of the system causing electric current by Faraday's effect. The harvesting device was optimized using finite element analysis (ANSYS software) and the output power was found to be $0.25 \mathrm{~W}$ from the electromagnetic mechanism and $0.25 \mathrm{~mW}$ using the vibration at $35 \mathrm{~g}$ vibration acceleration and $20 \mathrm{~Hz}$ frequency.

Regarding hybrid energy harvesting for thermal and mechanical energies conversion, some materials such as Lithium Niobate, PVDF, PZT, and $\mathrm{ZnO}$ are of interest as they have both the pyroelectric and piezoelectric properties together. Whilst the pyroelectric effect is used to convert the thermal energy into electrical one, the piezoelectric effect is used to convert the mechanical energy. Piezoelectric energy harvesting for scavenging ambiant mechanical vibration energies has raised a paramount of works in the literature. On the other hand the various studies on thermal energies scavenging have principally been carried out with the pyroelectric effect as an alternative method to the thermoelectric effect due to its high efficiency $[14,15,16$, 17]. The main need for the use of pyroelectric effect is the variation of the temperature other the time. In [18], Cottrill et al. applied an induced temperature change environment, the diurnal temperature variation in a desert, directly to a thermal energy harvesting system. In [19], Zhang et al. used a laser heat source for a thin film pyroelectric system. Some works were done in the absence of a heat sink, and consequently the harvester has low heat flux and low output power. To overcome this challenge, hybrid harvesting draws significant interest by coupling two or more effects. Most of the research works on hybrid thermal and mechanical energies harvesting can be classified into the following categories: research on new materials which could provide better properties for higher output power/energy [20,21], fabrication of a multilayered Nano generator for better performances [22,23] (various examples were reviewed in [21]), or proposal of a new mechanism functioning (bi-stable, multi-stable...) that can allow hybrid harvesting system $[24,25]$. To the authors best of knowledges, while there are several works consecrated to the structure design itself for vibrational energy harvesting [26, 27, 28, 29, 30], few works only exist for the design of strcutures for hybrid capability. For example, in [31] Gusarov et al. proposed polyvinylidene fluoride (PVDF) combined with shape memory alloys (SMA) as hybrid harvesting structure. A composite was laminated with the SMA on both sides of the PVDF polymer. Stress was generated by deformation due to the phase transition of SMA according to the change of temperature. As a result, the output voltage was improved by $77 \%$ on hybrid mode relative to when the harvester works only on thermal energy harvesting mode. It is worth to note that there exist hybrid mode (thermal and piezoelectric) for piezoelectric structures for actuation application [32] but they cannot be used for energy harvesting application, thus a complete redesign is required.

In this paper, we present an approach to hybrid harvesting that couples piezoelectric and pyroelectric effects simultaneously, harvessing waste heat and mechanical vibration energies from automobiles. The proposed structure is based on three materials (PVDF/Polyvinylidene fluoride piezoelectric, Lithium Niobate piezoelectric, and a SMA/Shape Memory Alloy materials) allowing an optimal functioning for the two energies to be harvested. First the paper presents the principle and the fabrication of the structure in section-2. Then, section-3 is devoted to theoretical analysis and formulation of the of the structure functioning. Finally section- 4 presents the finite elements simulation and experimental results.

\section{PRESENTATION OF THE HYBRID THERMAL PIEZOELECTRIC ENERGY HARVESTING}

\section{A. The principle of functioning and the structure}

In order to harvest the two forms of energy (vibrational and thermal), we propose cantilevers structures named as LSP (lithium niobate as the top layer, shape memory as middle layer and PVDF as the bottom layer), see Fig.1. The lithium niobate and PVDF are piezoelectric materials and are used for harvesting both the vibrational and thermal energy as they exhibit both the piezoelectric and pyroelectric effects. The SMA is used here to generate large strain and stress even in a narrow temperature variation range. More precisely, the SMA will induce and impose the shape memory effect to the LSP structure. As a consequence, the latter will be able to generate electric energy from temperature fluctuation based on two different energy conversion principles at once: (1) pyroelectric effect, (2) piezoelectric effect driven by the deformation imposed by the SMA.

As a summary, the output electrical energy/power is resulted by:

- the scanvenging of surrounding vibrational energy that was converted thanks to the piezoelectric effect of the PVDF and the lithium niobate materials,

- the scavenging of surrounding temperature variation that was converted into mechanical deformation/stress by the SMA material thanks to its shape memory effect, and which afterwards converted into the electrical energy thanks to the piezoelectric effect of the PVDF and the lithium niobate materials,

- and the scavenging of the surrounding temperature variation thanks to the pyroelectric effect of the PVDF and the lithium niobate materials.

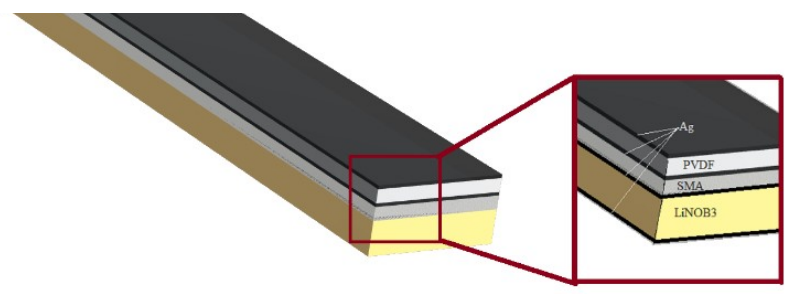

Figure 1: A Tri-Layered configuration of the cantilever LSP 


\section{B. Fabrication of the structure}

The fabrication process started by gluing the three different layers together: the PVDF polymer, the $\mathrm{LiNbO}_{3}$ (lithium niobate) ceramics, and the metal SMA (shape memory alloy: NITINOL 55:45 Ni: Ti) with dimensions specified in the table 1. The PVDF piezoelectric material was from Piezo Systems Inc, the SMA was from Nexametals and the lithium niobite was from Roditi systems Inc. First, the three materials having wide surfaces were glued together using silver glue and epoxy. To solidify the glue, the resulted trilayer plate is heated inside an oven at 100 degree Celsius during one hour and then cooled down. After that, a laser machine (Siro Lasertec GmbH, Pforzheim, Germany) is used to cut the plate and obtain several cantilevers following a CAD model.

The resulted prototype is showed in Fig.2. The figure presents the cantilever structure (tri layered) of the hybrid energy harvester itself, its wiring and support which was fabricated with $3 \mathrm{D}$ printing machine, a basis on which the platform is placed, and an oscilloscope that permits to observe the output voltage. Note that the support clamps the cantilever structure at one of its extremities. The fabricated cantilever has a resonance frequency of $286 \mathrm{~Hz}$, which is convenient to our application. Indeed, the range of vibration in our automobiles we would like to appky the harvester is from $1 \mathrm{~Hz}$ to $300 \mathrm{~Hz}$. In Fig. 3 the fabricated structure and the support are shown separately.

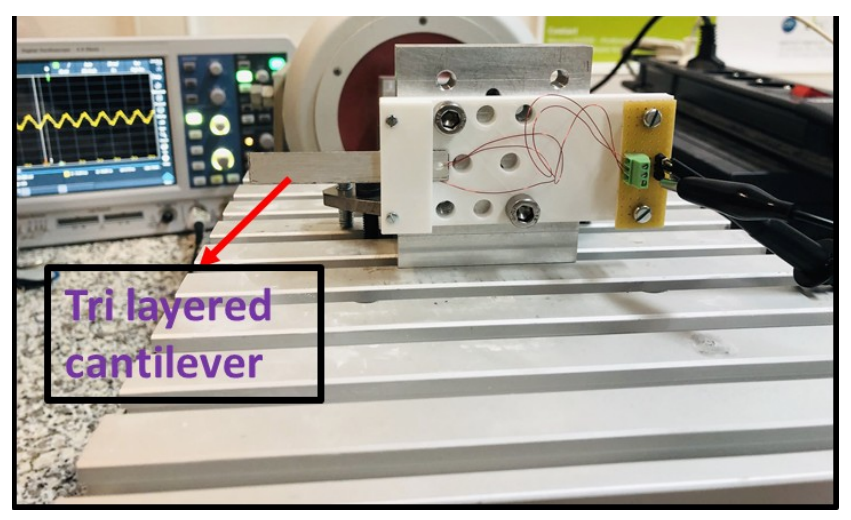

Figure 2: The prototype having tri layered configuration LSP
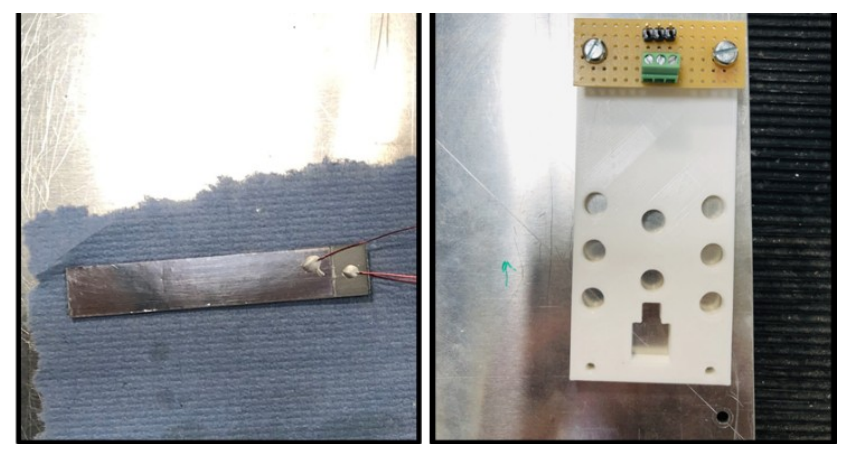

Figure 3: A) The final fabricated tri layer cantilever B) the $3 D$ printed support
Table 1: Dimensions of the energy harvesting cantilever.

\begin{tabular}{|l|l|l|l|l|l|}
\hline Materials & $\begin{array}{c}\mathrm{L}(\mathrm{mm}) \\
\text { length }\end{array}$ & $\begin{array}{c}\mathrm{b}(\mathrm{mm}) \\
\text { width }\end{array}$ & $\begin{array}{c}\mathrm{H}(\mathrm{mm}) \\
\text { thickness }\end{array}$ & $\begin{array}{c}\rho(\mathrm{g} / \mathrm{cm} 3) \\
\text { density }\end{array}$ & $\begin{array}{c}\mathrm{E}(\mathrm{Pa}) \\
\text { Young } \\
\text { modulus }\end{array}$ \\
\hline PVDF & 60 & 10 & 0.052 & 1.78 & 2 \\
\hline $\begin{array}{l}\text { Lithium } \\
\text { Niobate }\end{array}$ & 60 & 10 & 0500 & 4.865 & $1.7 \times 10^{11}$ \\
\hline SMA & 60 & 10 & 0.190 & 8.9 & $100 \times 10^{9}$ \\
\hline
\end{tabular}

\section{ANALYTiCAl EVALUATION}

\section{A. Energy harvesting from piezoelectric effect}

The electric charge generated by piezoelectric effect in a bending structure is obtained from:

$$
Q_{\text {piezo }}=\left(\mathrm{Fd}_{33} \mathrm{~L}_{\mathrm{p}}\right) / \mathrm{h}_{\mathrm{p}}
$$

where $F$ is the static force applied at the cantilever tip, $d_{33}$ is the piezoelectric strain coefficient, Lp is the length, and $\mathrm{hp}$ is the thickness of the piezo layer. The strain of the piezo layer is given by:

$$
\varepsilon_{p}=\frac{h_{p}}{2 R_{s}}
$$

Substituting the force $\mathrm{F}=\varepsilon_{p} E_{p} h_{p} b_{p}$ to equ. 3 results in:

$$
Q_{\text {Piezo }}=h p^{2} b_{p} d_{33} \frac{L_{p}}{2 R_{s}}
$$

where $\mathrm{E}_{\mathrm{P}}$ is the Young's modulus of the piezo layer.

Current can be calculated as follow:

$$
I_{\text {ріеzo }}=\alpha_{\text {piezo }} u-C V_{\text {piezo }}
$$

with $I_{\text {piezo }}$ outgoing current; $V_{\text {piezo }}$ voltage; u: displacement (tip bending of the cantilever); $\alpha_{\text {piezo }}$ : force (piezo) factor; $C$ : equivalent capacitance of the cantilever.

Assuming a harmonic excitation as surrounding vibration, the displacement of the piezo layers is:

$$
u=u_{M} \sin \left(\omega_{\text {Piezo }} t\right)
$$

with $\mathrm{u}_{\mathrm{M}}$ : displacement magnitude, $\omega_{\text {piezo }}=2 \pi f_{\text {piezo }}$ : frequency of excitation, $t$ : time.

The output energy extracted from the piezo layer can be calculated as:

$$
E_{\text {piezo }}=\frac{1}{2} C V_{\text {piezo }}^{2}
$$

while the output power is:

$$
P_{\text {piezo }}=f_{\text {piezo }} E_{\text {piezo }}
$$

where $f_{\text {piezo }}$ is the frequency of excitation.

Considering a short-circuit (the current $I_{\text {piezo }}=0$ in equ.6), we can evaluate the theoretical maximal output power by combining equ. 6 , equ. 8 and equ. 9 :

$$
P_{\text {piezo }}=f_{\text {piezo }} \frac{\alpha_{\text {piezo }^{2} U_{M^{2}}}}{2 C}
$$


Note that the above formulation is when we have one piezo layer. In our structure in which there are two piezo layers (PVDF and lithium niobate), the output power will be the sum of the two individual powers each of which is evaluated by the same equation than equ. 10 .

\section{B. Energy harvesting from pyroelectric effect}

The electric charge $\mathrm{Q}_{\text {pyro }}$ is given by:

$$
Q_{\text {Pyro }}=p A \Delta T
$$

where $p$ is the pyroelectric coefficient, $\mathrm{A}$ is the surface area of the pyro material and $\Delta T$ is the temperature variation. Therefore:

$$
Q_{\text {Pyro }}=\rho b L \Delta T
$$

The current can be calculated as follow:

$$
I_{\text {pyro }}=\alpha_{\text {pyro }} T-C V_{\text {pyro }}
$$

with $I_{\text {pyro }}$ : outgoing current; $V_{\text {pyro }}$ : voltage; T: temperature; $\alpha_{\text {pyro }}$ force (pyro) factor; C: equivalent capacitance.

Consider a harmonic temperature fluctuation such that:

$$
T=T_{M} \cos \left(\omega_{\text {pyro }} t\right)
$$

with $\mathrm{T}_{\mathrm{M}}$ : Temperature variation magnitude, $\omega_{\text {pyro }}=2 \pi f_{\text {pyro }}$ : pulsation, t: time,

The extracted energy for a pyroelectric effect is:

$$
E_{\text {pyro }}=\frac{1}{2} C V^{2}
$$

and the output power is:

$$
P_{\text {pyro }}=f_{\text {pyro }} E_{\text {pyro }}
$$

where $f_{\text {pyro }}$ is the frequency of excitation.

Considering a short-circuit (i.e. $I_{\text {pyro }}=0$ ), the maximal power one can extract can be obtained by combining equ.13, equ. 15 and equ. 16 :

$$
P_{\text {pyro }}=f_{\text {pyro }} \frac{\alpha_{p y r o^{2}\left[\sin (\beta) \mid T_{M^{2}}\right.}}{C}
$$

where $\beta$ is a conduction angle.

\section{Energy harvesting from both excitation}

The total voltage simultaneously obtained from the two different sources of energy is:

$$
V_{\text {total }}=V_{\text {piezo }}+V_{\text {pyro }}
$$

$\mathrm{V}_{\text {total }}$ : total voltage from both the sources, $\mathrm{V}_{\text {piezo }}$ is the piezoelectric voltage, $\mathrm{V}_{\text {pyro }}$ : pyroelectric voltage. Note that these voltages are obtained from equ. 6 and equ. 13 respectively when the currents are equal to zero for a maximal extraction:

$$
\begin{gathered}
V_{\text {piezo }}=\frac{\alpha_{\text {piezo }^{2} U_{M^{2}}}}{C} \\
V_{\text {pyro }}=\frac{\alpha_{\text {pyro } 2\left[\sin (\beta) \mid T_{M^{2}}\right.}}{C}
\end{gathered}
$$

Hence:

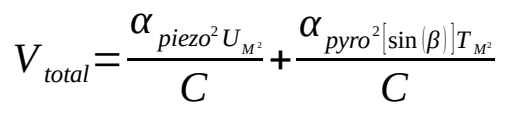

On the other hand, the total power from both sources is:

$$
P_{\text {total }}=P_{\text {piezo }}+P_{\text {pyro }}
$$

which results in:

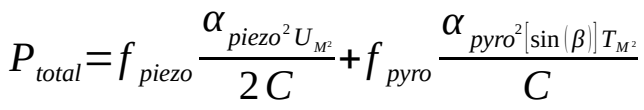

It is worth to note that the above output power is from one single piezo-pyro material. However, the proposed structure in this paper contains two piezo-pyro materials: PVDF and lithium niobate. Hence, each of them will exhibit a power depicted by equ. 22 and the final power will be the sum of them.

\section{SIMULATION AND EXPERIMENTAL RESULTS}

\section{A. Simulation results}

The aim of the simulation is to analysis the behavior and performances of the hybrid energy harvester by Comsol Multiphysics using finite element software. In the simulation, the analysis is however made individually for the thermal (pyro effect) and for the vibrational (piezo effect) functioning.

First, for the vibrational energy harvesting, knowing the resonance frequecy of the strcuture is important. Indeed the output energy/power will be maximized if the excitation frequency $f_{\text {piezo }}$ is equal or almost to the structure resonance frequency. By using Comsol Multiphysics software, a harmonic analysis was performed and shows a resonance frequency of the structure at $284 \mathrm{~Hz}$. Further experiment (see section-B) shows that the proposed structure has a resonance frequency of $286 \mathrm{~Hz}$ which is therefore near the simulation one. Then, by varying the excitation frequency from $1 \mathrm{~Hz}$ to in excess of $400 \mathrm{~Hz}$, and by connecting the harvester to the oscilloscope, we obtain the output voltage as depicted in Fig.4. It clearly indicates that the voltage is maximal at about $286 \mathrm{~Hz}$.

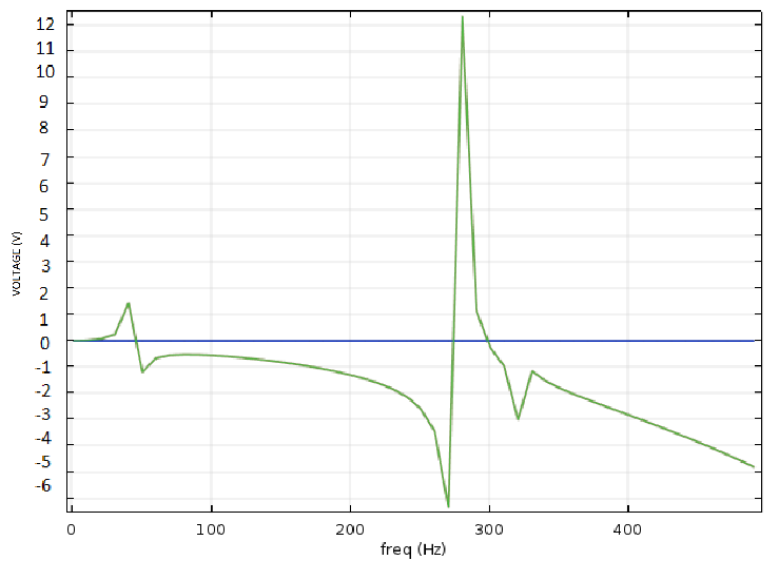

Figure 4: output voltage versus excitation frequency.

In order to analyse the mechanism functioning in thermal energy harvesting, finite element method simulation has also 
been performed using Comsol Multiphysics for the thermal effect. To this aim, we decided to use a closed system (like oven) in which we can design the heating-cooling cycle as per our wish, see the box depicted in Fig.5. The figure shows the positioning of the cantilever inside the oven and the maximum and minimum temperature inside it.

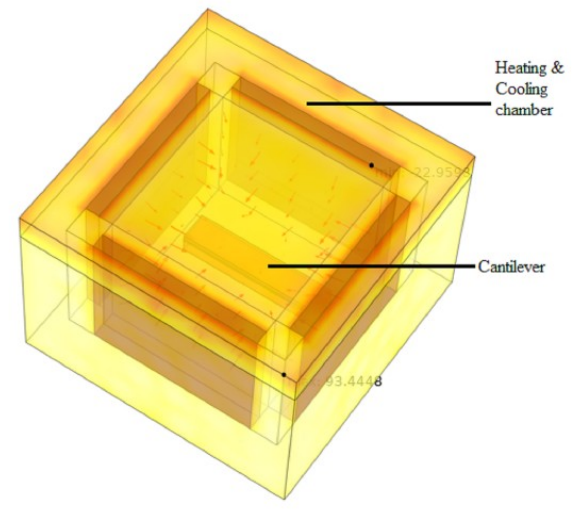

Figure 5: The cantilever inside a box (oven) and representation of the maximum and minimum temperatures inside it.

The simulation model consists of a combination of the heat transfer and laminar flow modules with the software. Temperature variation has been calculated along the cantilever for $600 \mathrm{~s}$ until steady oscillation was reached. Heat was transferred from a $120^{\circ} \mathrm{C}$ heat source through convection and conduction. The model is a pure structural mechanics problem. Then we calculated the thermal expansion of the material inside the environmental by using:

$$
\varepsilon_{t h}=d L\left(T, T_{\text {ref }}\right)
$$

where the thermal strain function is $\mathrm{dL}$, the reference temperature Tref $=20^{\circ} \mathrm{C}$, and $\mathrm{T}$ was the prescribed temperature. We also calculated the stress and the bending of the cantilever. Finally, we simulated the voltage generated by the cantilever considering only the pyro effect. Fig.6 shows the results of the voltage which goes beyond $6 \mathrm{~V}$ in absolute value.

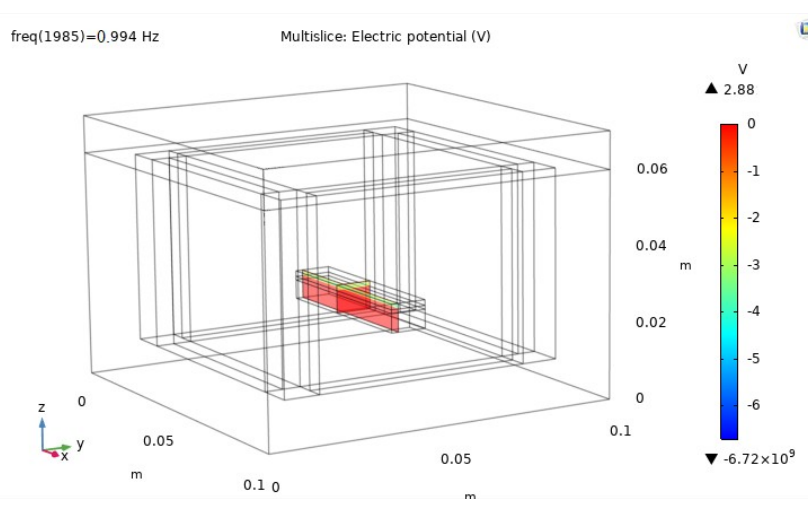

Figure 6: Calculated voltage for the cantilever inside the Oven.

\section{B. Experimental results}

To experimentally test the energy harvesting when vibration is the sole excitation, the cantilever structure was clamped to a shaker. The frequency and the torque/force magnitude generated by the shaker can be controlled through its panel. Under the vibrational excition from the shaker, the harvester produces AC electrical output. Then, the output signal from the harvester is connected to a resistive load. A 4-channel oscilloscope is used to perform real time measurements. The vibration frequency and its excitation magnitude are varied during the test. The maximum voltage or maximum power is used to evaluate the system performance. The harvester produces the maximum voltage when the excitation frequency is close to the structure resonance frequency. Fig.6 presents the results when using an excitation frequency of $290 \mathrm{~Hz}$, with which the output voltage is maximum and is equal to $12 \mathrm{~V}_{\mathrm{p}-\mathrm{p}}$.

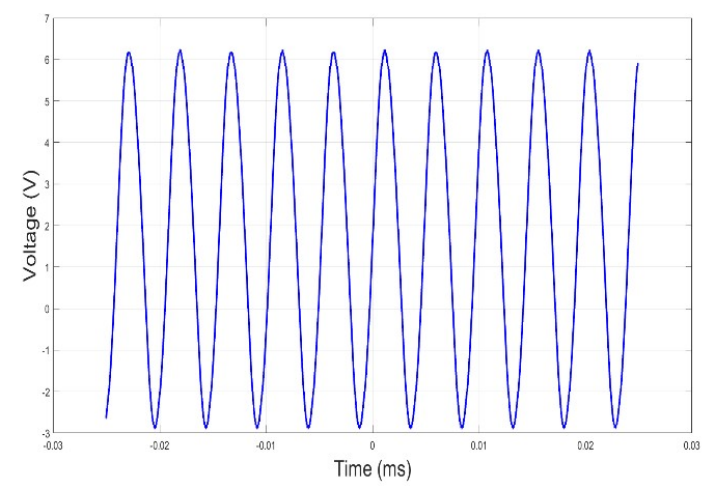

Figure 6. Experimental output voltage at $29 \mathrm{~Hz}$.

To experimentally test the thermal energy harvesting, an oven is used in which the temperature is set to fluctuate at approximately $0.2 \mathrm{~Hz}$. Fig. 10 shows the experiment results where the open circuit voltage is measured, as well as the temperature. The positive peaks of voltage indicate that the cantilever heats up (dT/dt $>0)$ after release. The negative peaks indicate that the cantilever is cooling down. However, at this time the output changes slowly, which is due to the pyroelectric effect. The enlarged figure shows a gradually increasing pyro electricity between sharp peaks during cooling, $(\mathrm{dT} / \mathrm{dt}<0)$. Finally, from the thermal part we were able to generate $3.2 \mathrm{~V}_{\mathrm{p}-\mathrm{p} \text {. }}$

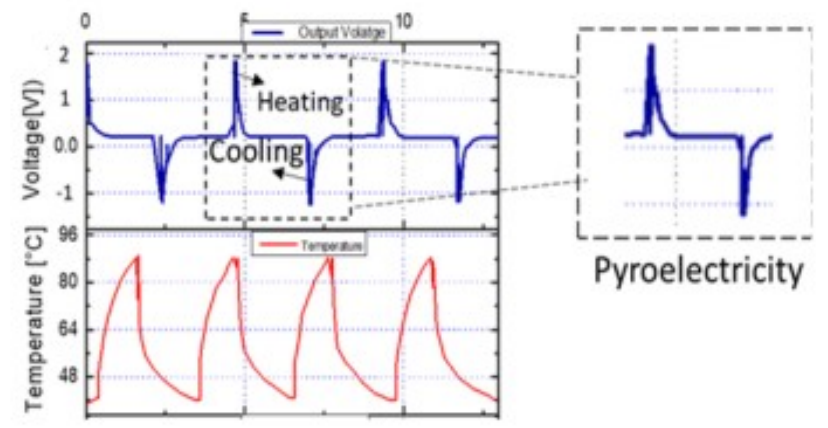

Figure 21: Open circuit voltage and temperature evolution.

\section{CONCLUSION AND PERSPECTIVES}

In the present paper, we presented a hybrid energy harvesting strcture that is able to convert mechanical vibrational energy and thermal energy from the same element. The structure consist of three different materials glued each other: two piezoelectric materials (PVDF polymer, lithium niobate) and one shape memory alloy. After giving an theoretical analysis of the functioning of the harvesting structure, we performed simulation and experimental tests. The results demonstrated that we were able to extract up tp $12 \mathrm{Vpp}$ when the excitation frequency is around $286 \mathrm{~Hz}$. On the other hand, we were able to extract 
$3 \mathrm{Vpp}$ when the thermal excitation is applied. Future works will consist in experimentally test the structure with both thermal and vibrational simultaneously. Perspective also includes optimization of the hybrid structure by using methodological approaches, such as topology optimization or interval techniques [33] already applied to piezoelectric actuators structures [34,35]. The aim is to find the appropriate and the optimal dimensions of the harvester such that one maximizes its performances. We also plan to analyze

\section{ACKNOWLEDGMENT}

This project has been supported by the European ITN Marie Curie ENHANCE project. Members of MACS research group at AS2M/FEMTO-ST are also acknowledged for their kind support as well as the Mechanical department of FEMTO-ST for some experiments. The authors are also grateful to the members of PRIMES center of technology and LGP laboratory, both of ENIT-INPT, for the support to extensive experimental works a part of which was also supported by the national ANR CODE-TRACK project.

\section{REFERENCES}

[1] M. Mark et al., Symp. on VLSI circuits, Kyoto, Japan, 15-17 June, pp 168-171, 2011.

[2] Radousky H B and Liang, H 2012 Nanotechnology 23502001.

[3] Y. Yang et al., 'Flexible hybrid energy cell for simultaneously harvesting thermal, mechanical, and solar energies'. ACS nano 7(1): 785-790, 2012.

[4] P. Regtien and E. Dertien, 'Piezoelectric sensors', in Sensors for Mechatronics, 2018.

[5] M. Rakotondrabe, 'Combining self-sensing with an Unkown-InputObserver to estimate the displacement, the force and the state in piezoelectric cantilevered actuator', American Control Conference, pp.4523-4530, Washington DC USA, June 2013.

[6] S. J. Nayar ate al., Novel wind/diesel/battery hybrid, 1993.

[7] H. Xue et al., "Broadband piezoelectric energy harvesting devices using multiple bimorphs with different operating frequencies", Ultrasonic, Ferroelectrics, and Frequency Control, IEEE Transactions on, 55(9), 2104-2108, 2008.

[8] G. Gonsalez et al., 'Energy Harvesting Using Piezoelectric and Electromagnetic Transducers', 9th Brazilian Conference on Dynamics, Control and Their Applications, Serra Negra, Brazil, p.1166-1171, 2010.

[9] M. Iqbal, 'Simulation of a small wind fuel cell hybrid energy system Renewable Energy', 28(4), April 2003, Pages 511-522, 2003.

[10] R. Challa et al.,'A coupled piezoelectric-electromagnetic energy harvesting technique for achieving increased power output through damping matching, Smart Materials and Struct, 18(9):095029. [doi:10.1088/0964-1726/18/9/095029, 2009.

[11] C. Dukhyun et al., 'Piezoelectric touch-sensitive flexible hybrid energy harvesting nano architectures', Nanotechnology 21405503 doi:10.1088/0957-4484/21/40/405503, 2010.

[12] T. Yonas et al., 'Multimodal Energy Harvesting System: Piezoelectric and Electromagnetic', Journal of Intelligent Material Systems and Structures, vol. 20 no. 5 625-632, 2009.

[13] G. Sebald et al., 'On thermoelectric and pyroelectric energy harvesting', Smart Mater Struct 2009;18(12):125006,

[14] J. Xie et al., 'Performance of thin piezoelectric materials for pyroelectric energy harvesting'. J Intell Mater Syst Struct2010;21(3):243-9, 2010.

[15] C.R. Bowen et al., 'Pyroelectric materials and devices for energy harvesting applications', Energy Environ, Sci2014;7(12):3836-56, 2009.
[16] I.M. McKinley et al., 'Novel thermomechanical energy conversion cycle', Appl Energy 2014;126:78-89, 2014.

[17] A.L. Cottrill et al., 'Persistent energy harvesting in the harsh desert environment using a thermal resonance device: Design, testing, and analysis', Appl Energy, 235:1514-23, 2019.

[18] Z. Zhang et al., 'Management and storage of energy converted via a pyroelectric heat engine', Appl Energy, 230:1326-31, 2018.

[19] U. Zhang Y, et al., 'Enhanced pyroelectric and piezoelectric properties of pzt with aligned porosity for energy harvesting applications', J Mater Chem A, 5(14):6569-80, 2017.

[20] Y. Tang et al., 'Enhanced pyroelectric and piezoelectric responses in W/Mn-codoped Bi4Ti3O12 aurivillius ceramics', J Eur Ceram Soc, 38(16):5348-53, 2018.

[21] J-H. Lee et al., 'Highly stretchable piezoelectric-pyroelectric hybrid nanogenerator', Adv Mater, 6(5):765-9, 2014.

[22] M-H. You et al., 'A self-powered flexible hybrid piezoelectricpyroelectric nanogenerator based on non-woven nanofiber membranes', J Mater Chem A, 6(8):3500-9, 2018.

[23] J-H. Lee et al., 'All-in-one energy harvesting and storage devices', J Mater Chem A, 4(21):7983-99, 2016.

[24] B. Gusarov et al., 'Thermal energy harvesting by piezoelectric pvdf polymer coupled with shape memory alloy', Sensors Actuat A: Phys, 243:175-81, 2016.

[25] M-S. Kim et al., 'Design of wearable hybrid generator for harvesting heat energy from human body depending on physiological activity', Smart Mater Struct; 26(9):095046, 2017.

[26] A. Homayouni, et al., 'Analytical Modelling and Optimization of a Piezoelectric Cantilever Energy Harvester with In-span Attachment', MDPI Micromachines, Special Issue on 'Piezoelectric Nanogenerators for Micro-Energy and Self-Powered Sensors', 11(6), pp.591, DOI:10.3390/mil 1060591, June 2020.

[27] A. Homayouni et al., 'Topology Optimization of 2DOF Piezoelectric Plate Energy Harvester Under External In-Plane Force', Springer Journal of Micro-Bio Robotics, DOI.10.1007/s12213-020-00129-0, March 2020.

[28] A. Homayouni et al., 'Multi directional piezoelectric plate Energy harvesters designed by topology optimization algorithm', IEEE Robotics and Automation Letters, DOI.10.1109/LRA.2019.2962367, 2020.

[29] T. Schlinquer et al., 'Optimal design of a unimorph piezoelectric cantilever devoted to energy harvesting to supply animal tracking devices', IFAC World Congress, pp.15165-15170, Toulouse France, July 2017.

[30] K. Rabenorosoa and M. Rakotondrabe, 'Performances analysis of piezoelectric cantilever based energy harvester devoted to mesoscale intra-body robot', SPIE - Sensing Technology+Applications; Sensors for Next Generation Robots conference , 9494-28, Baltimore Maryland USA, April 2015.

[31] D. Zakharov et al., 'Combined Pyroelectric, Piezoelectric and Shape Memory Effects for Thermal Energy Harvesting', IOP Power MEMS Journal of Physics: Conference Serie 476 012021, 2013.

[32] M. Rakotondrabe and A. Ivan, 'Development and dynamic modeling of a new hybrid thermo-piezoelectric micro-actuator', IEEE Transactions on Robotics, Vol.26, Issue.6, pp.1077-1085, December 2010.

[33] M. Rakotondrabe, 'Performances inclusion for stable interval systems', American Control Conference, pp.4367-4372, San Francisco CA USA, June-July 2011.

[34] S. Khadraoui et al., ' Optimal design of piezoelectric cantilevered actuators with guaranteed performances by using interval techniques', IEEE/ASME Transactions on Mechatronics, Volume 19, Issue 5, Page 1660-1668, October 2014.

[35] M. Rakotondrabe et al., 'Design of piezoelectric actuators with guaranteed performances using the performances inclusion theorem and interval tools', in 'Smart materials-based actuators at the micro/nano-scale: characterization, control and applications', Springer - Verlag, New York, ISBN 978-1-4614-6683-3, 2013. 